\title{
Modulation of Brain Activity after Learning Predicts Long-Term Memory for Words
}

\author{
Annika Hultén, ${ }^{1,2}$ Hannu Laaksonen, ${ }^{1}$ Minna Vihla, ${ }^{1}$ Matti Laine, ${ }^{2}$ and Riitta Salmelin ${ }^{1}$ \\ ${ }^{1}$ Brain Research Unit, Low Temperature Laboratory, Aalto University School of Science and Technology, FI-00076 Aalto, Finland, and ${ }^{2}$ Department of \\ Psychology and Logopedics, Åbo Akademi University, FI-20500 Turku, Finland
}

The acquisition and maintenance of new language information, such as picking up new words, is a critical human ability that is needed throughout the life span. Most likely you learned the word "blog" quite recently as an adult, whereas the word "kipe," which in the 1970s denoted stealing, now seems unfamiliar. Brain mechanisms underlying the long-term maintenance of new words have remained unknown, albeit they could provide important clues to the considerable individual differences in the ability to remember words. After successful training of a set of novel object names we tracked, over a period of 10 months, the maintenance of this new vocabulary in 10 human participants by repeated behavioral tests and magnetoencephalography measurements of overt picture naming. When namingrelated activation in the left frontal and temporal cortex was enhanced 1 week after training, compared with the level at the end of training, the individual retained a good command of the new vocabulary at 10 months; vice versa, individuals with reduced activation at 1 week posttraining were less successful in recalling the names at 10 months. This finding suggests an individual neural marker for memory, in the context of language. Learning is not over when the acquisition phase has been successfully completed: neural events during the access to recently established word representations appear to be important for the long-term outcome of learning.

\section{Introduction}

In neuroimaging studies on word learning and maintenance, the focus has been on short-term effects. These studies have mapped the influence of the type of information (phonological, orthographic, semantic, etc.) and the way it is encoded (deep or shallow, implicit or explicit, etc.) on the learning success (Demb et al., 1995; Gabrieli et al., 1996; Wagner et al., 1998; Heckers et al., 2002; Sandak et al., 2004; Breitenstein et al., 2005; Hultén et al., 2009). These types of word learning effects have most often been detected in the frontal cortex, interpreted as reflecting access to the new phonological form, together with activations in other regions, such as the temporal cortex, suggested to reflect phonological and semantic processing (Raboyeau et al., 2004; Sandak et al., 2004; Grönholm et al., 2005; Hultén et al., 2009). However, it is not known what happens after the active language learning period has ended, and how words acquire permanent representations.

The acquisition of new words and their successful maintenance necessarily involves the memory systems. Behavioral experiments have shown that word learning effects are observable either directly or indirectly up to one year after learning (Salasoo et al., 1985; Breitenstein and Knecht, 2002; Breitenstein et al.,

Received March 12, 2010; revised Aug. 16, 2010; accepted Sept. 17, 2010.

This work was supported by the Academy of Finland (Centre of Excellence Programme 2006 -2011, Neuro2005 Programme, Research Grant \#129160), the Finnish Cultural Foundation, the Sigrid Jusélius Foundation, and a Joint Committee for Nordic Research Councils for the Humanities and the Social Sciences grant for the Nordic Centre of Excellence in Cognitive Control.

Correspondence should be addressed to Annika Hultén, Low Temperature Laboratory, Aalto University, P.0. Box 15100, 00076 AALT0, Espoo, Finland. E-mail: annika@neuro.hut.fi.

DOI:10.1523/JNEUROSCI.1278-10.2010

Copyright $\odot 2010$ the authors $\quad 0270-6474 / 10 / 3015160-05 \$ 15.00 / 0$
2004; Tamminen and Gaskell, 2008), but offer no information about the underlying neural mechanisms. A few neuroimaging studies on word acquisition have used recall periods longer than $2 \mathrm{~h}$, even up to 4 weeks, but they have concentrated on changes related to learning rather than on maintenance mechanisms (Raboyeau et al., 2004; Grönholm et al., 2005; Hultén et al., 2009). Conversely, the handful of imaging studies with retention periods spanning several days or even weeks have not used novel words but pairs of familiar words or sentences to elucidate the neural correlates of efficient encoding in the long-term episodic memory (Bosshardt et al., 2005) or asymmetries between encoding and retrieval (Tulving et al., 1994). The neural substrates of episodic memory encoding and retrieval have been described (Paller and Wagner, 2002; Habib and Nyberg, 2008) but, so far, there have been no long-term maintenance studies on newly learned words.

The present study set off from a situation where participants had successfully learned native-language names to 50 pictures of old tools that are no longer familiar to the modern-day human. As reported earlier by Hultén et al. (2009), at this point namingrelated magnetoencephalography (MEG) recordings showed a systematic increase of left frontal and temporal activation during naming of the newly learned items compared with both the prelearning recording with the same items and the naming attempts on untrained items. The neural learning effect was evident at $300-800 \mathrm{~ms}$ after picture presentation, before the overt production of the object name.

\section{Materials and Methods}

Experimental procedure. The participants were 10 healthy native Finnishspeaking adults (21-30 years, five females). All individuals gave their informed consent to participate in the study which was approved by the 
local ethics committee. The stimuli were black-and-white line drawings of real but old or rarely used tools and utensils. The participants were trained on the real Finnish names of 50 of these objects until they mastered at least $98 \%$ of the names (category Name). As a control condition, the study included another set of 50 similar items for which no information was given (NoName) and pictures of 50 highly familiar items (Fam). During the training, all the stimuli in all the categories were presented an equal number of times, in a random order, thereby ensuring equal visual familiarity of all objects. A more detailed description of the training procedure can be found in the supplemental material, available at www. jneurosci.org (see also Hultén et al., 2009, for a comprehensive description and the full set of stimuli).

MEG recordings were performed on five different days. The first recording (fully learned) was done on the following day after reaching the training criterion. To track the forgetting process, we summoned the participants after 1 week (Post1wk), 4 weeks (Post4wk), 2 months (Post2mo), and 10 months (Post10mo).

In the MEG recordings, the participants performed a simple delayed picture naming task on all the object images (without any other information), presented in a randomized order. The picture was presented for $150 \mathrm{~ms}$ and followed by a blank screen for $850 \mathrm{~ms}$ after which a question mark prompted the participant to name the depicted object. If the participants did not know the name or had forgotten it, they were instructed to say aloud the generic word "object" (in Finnish "esine"). The behavioral success in producing the correct names was evaluated during each MEG recording (online measure) and, after each MEG recording, in a separate paper-and-pencil test, without any time limit (offline measure) (cf. Hultén et al., 2009).

Data analysis. In the MEG analysis, the focus was on the $1000 \mathrm{~ms}$ interval between picture onset (time 0 ) and the prompt for overt picture naming; the $200 \mathrm{~ms}$ interval preceding the stimulus onset served as the baseline. The MEG signals were averaged across trials, separately for each stimulus category (Name, NoName, Fam), low-pass filtered at $40 \mathrm{~Hz}$ and baseline corrected to the $200 \mathrm{~ms}$ interval immediately preceding the picture onset. Two separate categories for trials with correct and incorrect responses were further computed for those individuals who correctly named between 33 and $67 \%$ of the new names at the final recording (thus providing a reasonable number of trials for both the correct and incorrect category). These additional categories could be studied in six participants; the neural single-trial responses to the to-be-forgotten versus to-beremembered stimuli were computed for all recordings and statistically evaluated at the individual level.

MEG sensor-level areal mean signals are reported in the supplemental material, available at www.jneurosci.org. The spatially summed effects evident at the sensor level were decomposed into separable time courses of activation of distinct cortical areas with the help of source analysis, performed using established procedures (Salmelin et al., 1994; Hultén et al., 2009). The active neural populations were modeled as equivalent current dipoles (ECDs) that were brought together into a multi-ECD model in which the local activation strengths, as a function of time, were allowed to adjust to best account for the measured data. Each ECD represents a distinct dipolar field pattern, indicating local synchronous neural activation. The number of ECDs was determined on the basis of independent magnetic field patterns. See supplemental material (available at www.jneurosci.org) for a detailed description of the ECD analysis.

To visualize the sources anatomically, ECDs were displayed on individual magnetic resonance images. For group-level visualization, all ECDs were displayed on one participant's brain surface, using an elastic transformation. For group-level analysis, active brain areas in individual participants were grouped into six regions (occipitoparietal, left/right parietal, left temporal, left/right frontal), based on anatomical proximity and similarity in timing. A cortical region was included in the analysis if it was active in at least 6 participants.

Statistical analyses. The MEG measure of interest was the mean activation strength at 300-800 ms poststimulus (100-200 ms for the transient occipital activation). To minimize spurious variation that could appear in recordings performed on different days, the activations of interest, i.e., those evoked by the Name and NoName items, were baseline corrected to

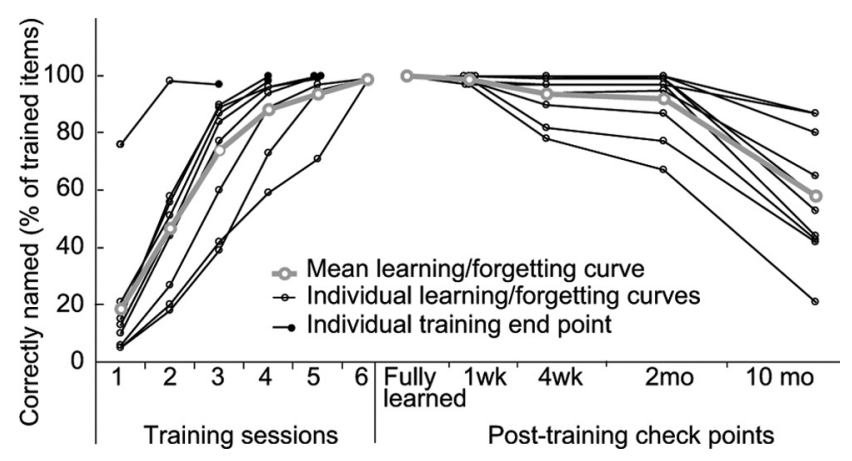

Figure 1. Behavioral learning and forgetting curves. Individual and mean curves. The learning rate was monitored with a questionnaire after each training session (offline measure). When the criterion of $98 \%$ correctly named objects was reached, the training was concluded (marked with a solid dot). An MEG recording was conducted on the following day (fully learned) and posttraining follow-up recordings at 1 week, 4 weeks, 1 month, and 10 months. The questionnaire was completed after each MEG recording.

the activation evoked by the Fam items within each recording, separately for each individual. This normalized measure was used in a grouplevel analysis using a repeated-measures $5 \times 2$ ANOVA (recording day $\times$ Name/NoName). Further correlation analyses in the brain regions showing significant effects were performed using Pearson's correlation coefficients. At the individual level, the stimulus effects were evaluated using a permutation test and by comparing the difference in signal strengths with the variation detected during the prestimulus baseline interval. For a more detailed description of the statistical analyses, see the supplemental material, available at www.jneurosci.org.

\section{Results}

\section{Behavior}

The learning phase of the study showed that the participants needed 3-6 daily training sessions to learn to name the 50 pictured objects (Hultén et al., 2009). The mean rate of acquisition was $21 \%$ (SD 5\%), i.e., 10 new names per training session (Fig. 1 ). The mean offline rate of forgetting was $-11 \%$ (SD $6 \%$ ), i.e., $\sim 5$ names per recording. The individual variation was much larger in the forgetting phase (especially in the last two recordings) than in the learning phase [training phase kurtosis $=1.4$, forgetting phase kurtosis $=-1.6]$. The learning rates were steeper than the forgetting rates $\left[t_{(8)}=3.3, p=0.011\right.$; rates corrected for directionality]. The individual learning rates did not correlate significantly with the maintenance of the new vocabulary 10 months posttraining $[r=0.6, p=0.103]$.

\section{Neural activation}

MEG was collected on a delayed picture naming task one day after the training criterion had been reached (fully learned) and at 1 week (Post1wk), 4 weeks (Post4wk), 2 months (Post2mo), and 10 months (Post10mo) posttraining. The cortical sequence of activation (Fig. 2) was comparable in all participants, advancing from the occipital cortex $(<200 \mathrm{~ms})$ to parietal $(200-400 \mathrm{~ms})$, temporal $(>200)$, and frontal $(>300)$ cortex, in line with earlier MEG studies of picture naming (Salmelin et al., 1994; Levelt et al., 1998; Vihla et al., 2006; Hultén et al., 2009; Liljeström et al., 2009). Activation was consistently detected in six regions, occipital $(n=10)$, left parietal $(n=7)$, right parietal $(n=10)$, left temporal $(n=9)$, left frontal $(n=8)$ and right frontal $(n=7)$ cortex (Fig. $2 a$ ). A few participants showed additional activation in the right temporal cortex, but this activation was not spatially or temporally consistent enough to justify group-level comparisons. The overall spatiotemporal sequence in each individual was 
a
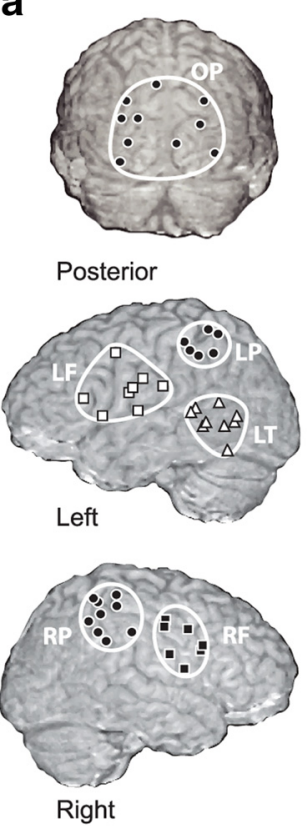

b

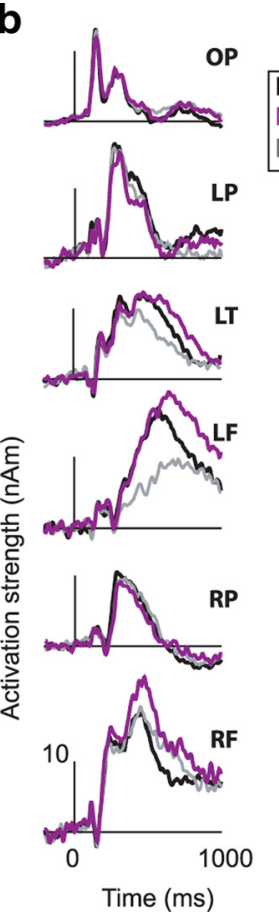

C
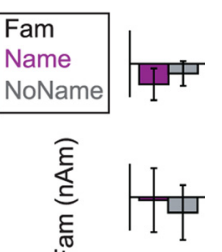

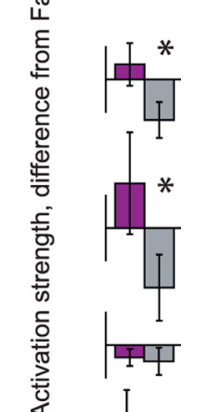

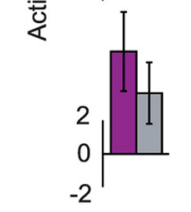

Figure 2. Neural effects of vocabulary acquisition and maintenance in a picture naming task, collapsed across all the five recording days. $\boldsymbol{a}$, Active brain areas in individual participants (dots represent the centers of active cortical patches) grouped into six regions [occipitoparietal (OP), left/right parietal (LP/RP), left temporal (LT), left/right frontal (LF/RF)]. $\boldsymbol{b}$, Mean time courses of activation for the Fam (black), correctly named Name (purple), and NoName (gray) stimuli in each region, pooled across all MEG recordings.c, The Name items evoked a significantly stronger activation (mean $\pm S E M)$ than NoName items in the left temporal and frontal regions $\left({ }^{*} p<0.05\right)$.

the same for all stimuli and for all MEG recordings but the local activation strengths varied (Fig. $2 b$ ).

In the left frontal and temporal region, responses evoked by the Name and NoName categories (baseline corrected to the Fam category) differed significantly from each other [left frontal: $F_{(1,6)}=9.0, p=0.024$; left temporal: $F_{(1,7)}=6.2, p=0.041$ ] (Fig. $2 c)$. In both regions, the Name items elicited stronger and the NoName items weaker activation than the Fam items across all recordings [mean (SD); left frontal: Name 2.7 (8.1), NoName -3.6 (5.3); left temporal: Name 0.9 (3.6), NoName -2.5 (3.0) $\mathrm{nA}$. There were no significant interactions between recording day and stimulus type in any region. The stronger activation to the Name than NoName items reached statistical significance in the individual participants as well (Fig. 3). The activation strength did not differ significantly between the to-be-forgotten and to-be-remembered items on any recording day, in any of the six participants in whom this comparison was possible.

The difference in activation strength between the Name and Fam items in the left frontal and temporal cortex over the course of the posttraining recordings showed considerable interindividual variation (Fig. 4a,b). Nevertheless, the first recall test 1 week posttraining should be a comparable situation across individuals, as the behavioral test indicated that all participants still remembered essentially all item names (online performance at post1wk, 97.8\%, SD 2.3). This session may thus serve as a critical test point where the new object names are retained in active vocabulary beyond immediate recollection. Interestingly, the individual change of neural activation from the fully learned to the Post1wk recording (Fig. 4a,b) covaried with the

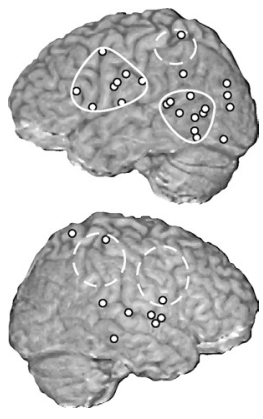

Figure 3. Significant stimulus effects in individual participants. The white dots indicate the source areas that showed stronger activation to the Name than NoName items in the fully learned condition and in three or more follow-up recordings. The white borders denote the group-level regions (Fig. 2) for reference. Both group-level and individual analyses indicated stimulus effects most consistently in the left temporal and frontal regions (solid white ellipses).

decline in online behavioral performance from the fully learned to the Post10mo recording (Fig. $4 c, d$ ). This correlation was significant for both the left frontal $[r=0.72, n=8, p=0.046]$ and left temporal activation change [ $r=0.76, n=9, p<0.018]$. Those individuals in whom the initially heightened neural activation to the Name items (with respect to the Fam items) remained unchanged or increased at 1 week posttraining retained a good command of the new names at 10 months. By contrast, the weaker the initial effect to new names had become at 1 week posttraining, the less successful were the participants in recalling the names at 10 months (Fig. $4 e, f$ ). In the most poorly performing participants, the activation to the Name items was reduced to the level of the NoName items in the Post10mo recording. The absolute level of neural activation in the fully learned recording did not correlate with the behavioral performance at Post10mo.

\section{Discussion}

The key finding in the present study was that a change of namingrelated neural activation in the left inferior frontal and posterior temporal cortex from the end of the learning phase to the recording 1 week later significantly predicted the access to the novel words as late as 10 months posttraining. It should be emphasized that these associations were not item-specific, as the cortical naming responses after learning were similar for the to-beremembered and to-be-forgotten words. Instead, these associations predicted which individuals maintained the newly learned names in their active vocabulary almost a year later.

Some earlier neuroimaging studies on memory have reported item-specific predictability of brain activity measures (Wagner et al., 1998; Habib and Nyberg, 2008). However, it may be that such associations are limited to the encoding phase, short retention intervals, and/or more episodic memory tasks. Our learning task tapped into the declarative memory (knowledge about facts and events) that maintains the network of word representations in the brain (Ullman, 2004). The items were all conceptually related: they were all man-made tools, real usable instruments with real Finnish names, and the participants were aware of this general context. This may have triggered the use of more global recall features for this set of items. Item-level effects were also lacking in the within-session comparisons of the MEG responses to remembered versus forgotten item names. This similarity may be due to the overlap in cognitive-linguistic operations: in both instances, object recognition was initiated, output phonology was activated, and articulation was programmed either for the object's name or, 


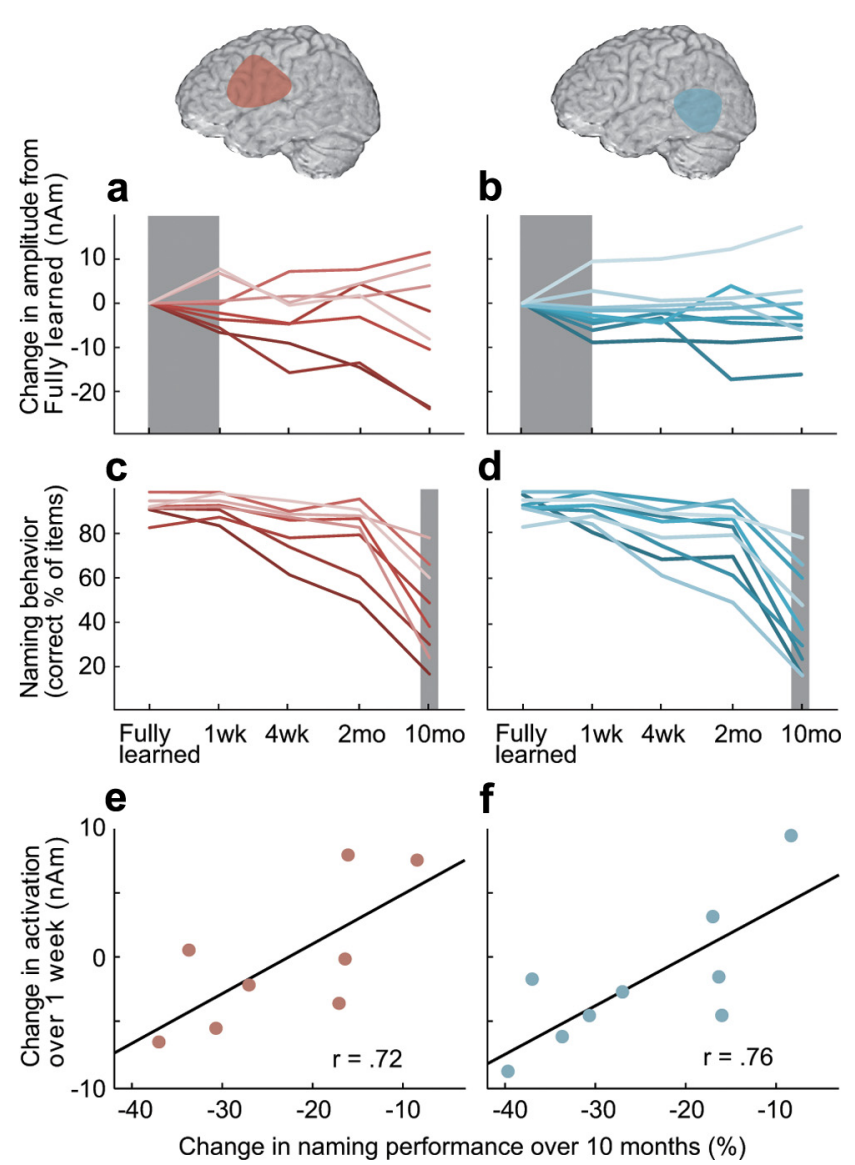

Figure 4. Correspondence between neural and behavioral measures of maintenance and forgetting. The behavioral measure was collected during the MEG recording (online). $\boldsymbol{a}, \boldsymbol{b}$, The relative change in the mean strength of activation to the Name items (after baseline correction to the Fam condition) with respect to the fully learned recording (zero level) in the left frontal $(\boldsymbol{a})$ and left temporal cortex $(\boldsymbol{b})$. The individual participants are denoted with different shades. The gray box marks the interval from the fully learned recording to the first follow-up recording 1 week later (Post1wk). $\boldsymbol{c}$, d, The drop in the naming performance during the MEG experiment from the fully learned to the final Post10mo recording (gray box), with the individual participants marked by different shades that correspond to the activation curves in the left frontal ( $\boldsymbol{a}$ vs $\boldsymbol{c}$ ) and left temporal cortex ( $\boldsymbol{b}$ vs $\boldsymbol{d}$ ). The behavioral rate of forgetting was quantified as the mean number of online errors across the multiple sessions within one MEG recording. $\boldsymbol{e}, \boldsymbol{f}$, The change of activation to the Name items from the fully learned to the Post1wk recording in the left frontal $(\boldsymbol{e})$ and temporal cortex $(\boldsymbol{f})$ covaried with the eventual decline in the online naming performance at the Post10mo recording.

if the search had failed to converge on a specific word, for the generic name "esine" (object).

The neural effects predicting long-term maintenance of new active vocabulary were found in well known parts of the brain's language and memory networks, namely the left posterior temporal and inferior frontal cortices. In language production, these areas have been implicated in both phonological and semantic processing (Salmelin et al., 1994; Bookheimer, 2002; Indefrey and Levelt, 2004; Vigneau et al., 2006). In studies focusing on memory systems, the left frontal lobe has been assigned a role in phonological working memory and semantic memory retrieval (for review, see Cabeza and Nyberg, 2000), whereas the declarative memory system is thought to be subserved mainly by the temporal lobes, with initial learning dependent on medial temporal lobe structures and long-term maintenance (as tested here) on the temporal cortex (Ullman, 2004).

The same cortical networks seem to be engaged in both the acquisition and long-term maintenance of words: The left infe- rior frontal and posterior temporal cortices showed the most pronounced overall learning effects, i.e., increased activation to the newly learned names at the fully learned recording (Hultén et al., 2009). Retrieving the trained names did not activate any additional areas at any of the postlearning recordings. The enhanced activation to the learned items cannot be accounted for by solely perceptual factors or recognition memory, as the control pictures (the NoName and Fam items) were encountered equally often and they were visually comparable (Hultén et al., 2009). The participants' high accuracy levels at phonological and semantic categorization tasks presented at the fully learned stage (reported in Hultén et al., 2009) further indicated that the words had been successfully incorporated in the mental vocabulary.

What could be the mechanisms underlying the association that we observed between the change in neural activation over 1 week after learning and the behavioral performance 10 months later? While this intriguing phenomenon requires further study, we can entertain some possible accounts. First, consolidation of the newly acquired lexical items might have occurred largely automatically (i.e., independent of active recollection or repetition), e.g., during sleep (Fenn et al., 2003; Davis et al., 2009), and be reflected as increased left temporal and frontal activation at Postlwk in those participants in whom this physiological process was more efficient. Second, one might also argue that the good long-term learners' enhanced brain responses were not based on automatic mechanisms but rather on stronger attention and motivation. However, such effects should be evident already during the learning phase and thus reflected in the behavioral learning rate or in the neural activation at the fully learned stage. Yet neither of these measures correlated with the long-term maintenance of the new words. A third alternative is that the active recall at the Post1wk recording served as a system-level reconsolidation stage that either reinforced or weakened the long-term memory trace. Animal studies have demonstrated that if the consolidation period is chemically interrupted, short-term memories do not enter the long-term store. Interestingly, it has further been shown that when long-term memories are reactivated they enter a state of instability, during which they must be reconsolidated to persist in the long-term store (Nader and Hardt, 2009). The present results might thus be a manifestation of the same basic organizational principle at the human cortical level for a complex cognitive task, linguistic processing.

The present MEG study describes a neural marker that relates to individual maintenance of new linguistic knowledge on the long term. This neural marker may reflect early reinforcement of newly learned words that is important for their persistence in memory. Knowledge of the neural underpinnings of how our brain transforms new words into easily accessible permanent representations can give valuable clues to more efficient language teaching and rehabilitation of language disorders. The current results are a potentially important step on this path, though further studies bridging the language and memory domains are needed to examine the specific factors that lead to the successful reinforcement of novel vocabulary.

\section{References}

Bookheimer S (2002) Functional MRI of language: new approaches to understanding the cortical organization of semantic processing. Annu Rev Neurosci 25:151-188.

Bosshardt S, Degonda N, Schmidt CF, Boesiger P, Nitsch RM, Hock C, Henke K (2005) One month of human memory consolidation enhances retrievalrelated hippocampal activity. Hippocampus 15:1026-1040.

Breitenstein C, Knecht S (2002) Development and validation of a language 
learning model for behavioral and functional-imaging studies. J Neurosci Methods 114:173-179.

Breitenstein C, Kamping S, Jansen A, Schomacher M, Knecht S (2004) Word learning can be achieved without feedback: implications for aphasia therapy. Restor Neurol Neurosci 22:445-458.

Breitenstein C, Jansen A, Deppe M, Foerster AF, Sommer J, Wolbers T, Knecht S (2005) Hippocampus activity differentiates good from poor learners of a novel lexicon. Neuroimage 25:958-968.

Cabeza R, Nyberg L (2000) Imaging cognition II: An empirical review of 275 PET and fMRI studies. J Cogn Neurosci 12:1-47.

Davis MH, Di Betta AM, Macdonald MJ, Gaskell MG (2009) Learning and consolidation of novel spoken words. J Cogn Neurosci 21:803-820.

Demb JB, Desmond JE, Wagner AD, Vaidya CJ, Glover GH, Gabrieli JD (1995) Semantic encoding and retrieval in the left inferior prefrontal cortex: a functional MRI study of task difficulty and process specificity. J Neurosci 15:5870-5878.

Fenn KM, Nusbaum HC, Margoliash D (2003) Consolidation during sleep of perceptual learning of spoken language. Nature 425:614-616.

Gabrieli JDE, Desmond JE, Demb JB, Wagner AD, V. SM, Vaidya CJ, Glover GH (1996) Functional magnetic resonance imaging of semantic memory processes in the frontal lobes. Psychol Sci 7:278-283.

Grönholm P, Rinne JO, Vorobyev V, Laine M (2005) Naming of newly learned objects: a PET activation study. Cogn Brain Res 25:359-371.

Habib R, Nyberg L (2008) Neural correlates of availability and accessibility in memory. Cereb Cortex 18:1720-1726.

Heckers S, Weiss AP, Alpert NM, Schacter DL (2002) Hippocampal and brain stem activation during word retrieval after repeated and semantic encoding. Cereb Cortex 12:900-907.

Hultén A, Vihla M, Laine M, Salmelin R (2009) Accessing newly learned names and meanings in the native language. Hum Brain Mapp 30:976-989.

Indefrey P, Levelt WJ (2004) The spatial and temporal signatures of word production components. Cognition 92:101-144.

Levelt WJ, Praamstra P, Meyer AS, Helenius P, Salmelin R (1998) An MEG study of picture naming. J Cogn Neurosci 10:553-567.

Liljeström M, Hultén A, Parkkonen L, Salmelin R (2009) Comparing MEG and fMRI views to naming actions and objects. Hum Brain Mapp 30:1845-1856.

Nader K, Hardt O (2009) A single standard for memory: the case for reconsolidation. Nat Rev Neurosci 10:224-234.

Paller KA, Wagner AD (2002) Observing the transformation of experience into memory. Trends Cogn Sci 6:93-102.

Raboyeau G, Marie N, Balduyck S, Gros H, Démonet JF, Cardebat D (2004) Lexical learning of the English language: a PET study in healthy French subjects. Neuroimage 22:1808-1818.

Salasoo A, Shiffrin RM, Feustel TC (1985) Building permanent memory codes: codification and repetition effects in word identification. J Exp Psychol Gen 114:50-77.

Salmelin R, Hari R, Lounasmaa OV, Sams M (1994) Dynamics of brain activation during picture naming. Nature 368:463-465.

Sandak R, Mencl WE, Frost SJ, Rueckl JG, Katz L, Moore DL, Mason SA, Fulbright RK, Constable RT, Pugh KR (2004) The neurobiology of adaptive learning in reading: a contrast of different training conditions. Cogn Affect Behav Neurosci 4:67-88.

Tamminen J, Gaskell MG (2008) Newly learned spoken words show longterm lexical competition effects. Q J Exp Psychol 61:361-371.

Tulving E, Kapur S, Craik FI, Moscovitch M, Houle S (1994) Hemispheric encoding/retrieval asymmetry in episodic memory: positron emission tomography findings. Proc Natl Acad Sci U S A 91:2016-2020.

Ullman MT (2004) Contributions of memory circuits to language: the declarative/procedural model. Cognition 92:231-270.

Vigneau M, Beaucousin V, Herve PY, Duffau H, Crivello F, Houdé O, Mazoyer B, Tzourio-Mazoyer N (2006) Meta-analyzing left hemisphere language areas: phonology, semantics, and sentence processing. Neuroimage 30:1414-1432.

Vihla M, Laine M, Salmelin R (2006) Cortical dynamics of visual/semantic vs phonological analysis in picture confrontation. Neuroimage 33:732-738.

Wagner AD, Schacter DL, Rotte M, Koutstaal W, Maril A, Dale AM, Rosen BR, Buckner RL (1998) Building memories: remembering and forgetting of verbal experiences as predicted by brain activity. Science 281: $1188-1191$. 